\title{
Reposição eletrolítica sobre variáveis fisiológicas de cavalos em provas de enduro de 30 e $60 \mathrm{~km}$
}

\author{
Electrolyte reposition on physiologic variables of horses submitted to 30 and $60 \mathrm{~km}$ endurance rides
}

\author{
Antônio Raphael Teixeira-Neto ${ }^{1}$ Guilherme de Camargo Ferraz ${ }^{1}$ Maria Isabel Mataqueiro ${ }^{2}$ \\ José Correa de Lacerda-Neto ${ }^{3}$ Antonio de Queiroz-Neto ${ }^{4}$
}

\section{RESUMO}

O presente estudo teve como objetivo a comparação de variáveis fisiológicas (freqüência cardíaca, peso corporal, hematócrito, proteínas totais) entre um grupo de eqüinos atletas submetidos a provas de enduro eqüestre nas distâncias de 30 e $60 \mathrm{~km}$ com velocidade média de 10 e $15 \mathrm{~km} \mathrm{~h}^{-1}$, respectivamente. Em ambas as provas, os animais foram divididos em dois grupos experimentais que receberam, ou não, reposição eletrolítica por via oral, na forma de pasta, antes, durante e após as referidas provas. $O s$ resultados obtidos revelaram que a administração da pasta eletrolítica não apresentou nenhum efeito colateral aos animais, nas distâncias em que foi avaliada, e sugerem que um suplemento à base de eletrólitos pode contribuir para o desempenho de cavalos submetidos a esforços prolongados.

Palavras chave: eletrólitos, eqüinos, enduro, parâmetros fisiológicos.

\section{ABSTRACT}

The objective of this work was to compare physiologic parameters of a group of trained horses submitted to 30 and $60 \mathrm{~km}$ endurance exercises with an average speed of 10 and $15 \mathrm{~km} / \mathrm{h}$, respectively. One of the groups was treated, orally, with an hipertonic electrolyte paste before, during and after the task. The results revealed that the administration of the electrolyte paste did not show any deleterious effect on the animals, and also suggest that the supplementation with an electrolyte source should contribute for the performance of horses submitted to prolonged efforts.

Key words: electrolytes, endurance, equine, physiologic values.

\section{INTRODUÇÃO}

O enduro é uma modalidade de esporte eqüestre caracterizada por um esforço aeróbico prolongado, de intensidade variável em que o cavalo é submetido a um trabalho permanente que muito exige dos sistemas orgânicos para que seja mantida a homeostasia. Dentre as funções fisiológicas destacase a importância da termorregulação corporal que é mantida, no cavalo, às expensas de fluidos corporais por meio da sudorese. A manutenção do equilíbrio hidroeletrolítico é de fundamental importância para a homeostase do organismo eqüino. Tomando-se por referência as perdas de peso corporal durante esforço de baixa intensidade e longa duração, estima-se que um cavalo perde, por hora, entre 10 e 15 litros de suor (CARLSON, 1985), contendo grandes concentrações de eletrólitos (KERR \& SNOW, 1982; CARLSON, 1983; ROSE et al., 1990).

O suor é o mecanismo primário de dissipação de calor tanto em indivíduos da espécie eqüina como em humanos (BURCH, 1945; HODGSON, 1994; McCONAGHY, 1994). Alterações no estado de hidratação podem influenciar o controle termorregulatório do suor. Este efeito é mediado por alterações na osmolalidade plasmática ao invés de trocas no volume plasmático (NIELSEN et al., 1971).

O suor eqüino, diferentemente do suor humano, é isotônico para alguns íons (e.g. sódio) e

\footnotetext{
${ }^{1}$ Médico Veterinário, Aluno de Pós-graduação, Faculdade de Ciências Agrárias e Veterinárias (FCAV), Universidade Estadual Paulista, Jaboticabal (UNESP).

${ }^{2}$ Biólogo, Técnico do Laboratório de Farmacologia, FCAV, UNESP.

${ }^{3}$ Médico Veterinário, Professor Assistente Doutor, Departamento de Clínica e Cirurgia, FCAV, UNESP.

${ }^{4}$ Médico Veterinário, Professor Adjunto do Departamento de Morfologia e Fisiologia Animal, FCAV, UNESP, Via de Acesso Prof. Paulo Donato Castellane, s/n, 14884-900, Jaboticabal, SP, Brasil. E-mail: aqueiroz@fcav.unesp.br. Autor para correspondência.
} 
hipertônico para outros (cloro e potássio), em relação ao plasma (McCONAGHY et al., 1995; McCUTCHEON et al., 1995; McCUTCHEON \& GEOR, 1996a). Diferenças na metodologia de coleta de suor em eqüinos explicam as altas concentrações de íons relatadas em estudos anteriores (SNOW et al., 1982; KERR \& SNOW, 1983; McCUTCHEON \& GEOR, 1996). Concentrações iônicas do suor são, em grande extensão, um reflexo da taxa de suor e, conseqüentemente, estão sujeitas a alterações baseadas nas condições ambientais e intensidade do exercício.

SCHOTT \& HINCHCLIFF (1993, 1998) concordaram que a depleção de fluidos corporais e das reservas de eletrólitos, como conseqüência da sudorese, representa uma limitação importante para a performance contínua durante exercícios prolongados de enduro. Distúrbios substanciais na composição dos fluidos corporais durante exercícios exaustivos também levam a um aumento no risco de lesões e vários problemas clínicos (JOHNSON, 1998).

A função dos eletrólitos no organismo animal é múltipla, pois não existe praticamente nenhum processo metabólico que seja independente ou mantenha-se inalterado diante de alterações na concentração de eletrólitos (FAN et al., 1994) que, no organismo como um todo, possuem a função principal de manutenção das forças osmóticas possibilitando o equilíbrio de líquidos entre os compartimentos intra e extracelulares. Na célula, desempenham funções básicas, tais como, condução nervosa e despolarização de fibras musculares, tornando possível a contração muscular. A extensão na qual essas funções podem ser prejudicadas em um dado déficit de eletrólitos induzido pelo exercício e na qual a capacidade atlética pode ser reduzida, permanece sem compreensão. Grandes reduções hidroeletrolíticas durante o exercício estão implicados no desenvolvimento da síndrome de exaustão e outros distúrbios metabólicos tais como o fluter diafragmático sincrônico (MANSMANN, 1974; HINTON, 1978). Portanto, a suplementação eletrolítica é importante pois restaura a função celular e ajuda a manter o equilíbrio de fluidos corporais durante o exercício.

$\mathrm{Na}$ tentativa de postergar a fadiga ou prevenir conseqüências potencialmente desastrosas decorrentes da exaustão, fluidos e eletrólitos são comumente administrados a cavalos atletas (SCHOTT \& HINCHCLIFF, 1993). Segundo SOSALEÓN (1998), quando cavalos são exercitados por um período prolongado, torna-se necessária a reposição de dois elementos chave: água e eletrólitos (principalmente sódio, potássio, e cloro). Em provas de enduro, água e eletrólitos podem e devem ser administrados durante o exercício, particularmente nos pontos de checagem veterinária ("check-points"), destinados à avaliação clínica dos animais no transcorrer da prova.

Os cavalos que desenvolveram a síndrome de exaustão também apresentaram aumentos pronunciados na concentração de proteína plasmática (CARLSON \& OCEN, 1979; ROWEL, 1983), devido à severa desidratação, relacionando-se a falhas nas trocas compensatórias entre os fluidos dos compartimentos intra e extracelulares. Esta desidratação durante o exercício de enduro causa uma marcante elevação na concentração de proteína plasmática, que está diretamente relacionada com déficit de sódio (CARLSON, 1983).

Durante o exercício de enduro, o cavalo apresenta um aumento no hematócrito devido a uma hemoconcentração associada à desidratação e também a um maior aporte de eritrócitos na corrente sangüínea em decorrência da contração esplênica que ocorre nesta espécie (JOHNSON, 1998). Entretanto, no repouso, cavalos de enduro tendem a ter valores de hematócrito menores que, quando comparados com aqueles treinados para corridas rápidas e curtas. Estes baixos valores são tidos como reflexo de um maior volume plasmático desenvolvido como a resposta ao treinamento para prolongadas perdas de fluidos e estresse do calor (CARLSON \& OCEN, 1979).

A administração de suplementos eletrolíticos apropriados pode ser benéfica na redução da incidência da síndrome de rabdomiólise eqüina (HARRIS \& SNOW, 1992). SCHOTT II et al. (1999) recomendam a administração de pastas eletrolíticas hipertônicas para cavalos de enduro na medida em que estes tenham freqüente acesso a água e sejam monitorados para beber continuamente. Estudos em atletas humanos de exercícios de longa duração demonstram melhor manutenção de fluidos corporais e aumento de desempenho com soluções reidratantes com maior osmolalidade e conteúdo de sódio quando comparados a soluções eletrolíticas hipo ou isotônicas, mais comumente utilizadas (GREENLEAF et al., 1998).

O presente estudo, teve como objetivo a discussão comparativa de parâmetros físiológicos (freqüência cardíaca, proteínas plasmáticas totais, hematócrito, hemograma) de um grupo de cavalos atletas, treinados sob um mesmo programa de condicionamento físico, para um mesmo tipo de esforço (enduro), submetidos ou não a uma reposição eletrolítica; antes, durante e depois do esforço, em condições experimentais que se assemelham a provas de enduro de 30 e 60 quilômetros. 


\section{MATERIAL E MÉTODOS}

No presente trabalho, foram realizadas duas provas de enduro de 30 e $60 \mathrm{~km}$ de extensão. Na primeira prova, para completar o percurso, os conjuntos percorreram dois anéis de 20 e $10 \mathrm{~km}$ de extensão, com velocidade média de $10 \mathrm{~km} \mathrm{~h}^{-1}$. Na segunda prova, de $60 \mathrm{~km}$, os conjuntos percorreram os mesmos anéis de 20 e $10 \mathrm{~km}$ de distância, porém a uma velocidade média de $15 \mathrm{~km} \mathrm{~h}^{-1}$ e um anel adicional de $30 \mathrm{~km}$ de extensão, a $10 \mathrm{~km} \mathrm{~h}^{-1}$

Na primeira prova de enduro $(30 \mathrm{~km})$, foram utilizados 15 animais ( 8 machos e 7 fêmeas), com peso variando de 346 a 464kg. Desses animais, 13 eram Puro Sangue Árabe (PSA) e 2 Mangalarga. Nesta etapa 8 animais receberam a pasta de eletrólitos (tratados) e 7 não (controle).

$\mathrm{Na}$ segunda prova de enduro $(60 \mathrm{~km})$, foram utilizados 18 animais (10 machos e 8 fêmeas), com peso variando de 340 a 440kg. Desses animais, 15 eram PSA, dois Mangalarga e um sem raça definida (SRD). Nesta etapa, oito animais foram tratados e dez formaram o grupo controle. Todos os animais utilizados nas duas provas foram considerados adultos, com idade variando entre 8,6 $\pm 3,3$ anos.

Os animais foram condicionados por um período de três e nove meses antes de serem submetidos aos testes de esforço de 30 e $60 \mathrm{~km}$, respectivamente. O condicionamento consistia em se montar os animais de três a cinco vezes por semana em trilhas demarcadas no Campus da Faculdade de Ciências Agrárias e Veterinárias (FCAV/UNESP) de Jaboticabal. Este trabalho era realizado por alunos de graduação e do Colégio Técnico Agrícola desta instituição, com peso médio de $55 \mathrm{~kg}$.

Os índices meteorológicos utilizados neste trabalho foram extraídos de um conjunto de dados pertencentes ao acervo da área de Agrometeorologia do Departamento de Ciências Exatas. Na primeira prova, a temperatura ambiente variou entre 20 e $29,5^{\circ} \mathrm{C}$ com a umidade relativa entre 32 e $64 \%$. Já na segunda prova tais dados variaram entre 26,3 e $32,5^{\circ} \mathrm{C}$ e 33 e $67 \%$, respectivamente (Tabela 1 ).

Os parâmetros fisiológicos e o sangue para os exames laboratoriais foram coletados nos seguintes momentos. Antes do início da prova $\left(\mathrm{M}_{0}\right)$, durante a primeira avaliação veterinária (chec-vet) na chegada do primeiro anel da prova (M ), durante a segunda chec-vet na chegada do segundo anel da prova $\left(\mathrm{M}_{2}\right)$; e no $\mathrm{M}_{3}$, referente a chegada do anel adicional, da prova de $60 \mathrm{~km}$. Todos os anéis eram concluídos no hospital veterinário. Ao completar um anel, o animal permanecia por 30 minutos na área de avaliação veterinária (chec-vet) até a largada para o próximo anel. As variáveis fisiológicas avaliadas foram: frequiência cardíaca, freqüência respiratória, coloração de mucosas, tempo de perfusão capilar e evidência ou não de claudicação. Foi avaliado também o peso do animal no decorrer da prova durante as checagens veterinárias.

Para a reposição oral de eletrólitos, utilizouse de uma pasta ${ }^{a}$ composta de $21 \mathrm{~g}$ de $\mathrm{NaCl}, 21 \mathrm{~g}$ de $\mathrm{KCl}, 4 \mathrm{~g}$ de $\mathrm{CaCl}_{2}$, 6,5g de $\mathrm{MgO}$, em excipiente qsp. $60 \mathrm{~g}$ e essência artificial de "tuti-fruti", que era administrada aos animais uma hora antes da prova, a cada $10 \mathrm{~km}$ de trilha e ao final de cada prova.

A colheita das amostras de sangue era realizada por meio de punção da veia jugular, com agulha $40 \times 12$ e com tubos a vácuo ${ }^{\text {b }}$ para retirada de um volume de $15 \mathrm{ml}$ em frascos com e sem anticoagulante (EDTA). Para o eritrograma, foi utilizado sangue contendo EDTA. As amostras foram analisadas através do contador automático ${ }^{c}$. A contagem diferencial de leucócitos foi realizada em extensões sangüíneas coradas pancromicamente, segundo a técnica preconizada por Rosenfeld (1947).

Para a análise estatística, utilizou-se programa computacional $^{\mathrm{d}}$. As médias dos efeitos da administração eletrolítica e momento foram comparados pelo teste de Tukey $(\mathrm{P}<0,05)$.

\section{RESULTADOS E DISCUSSÃO}

No presente estudo, foi observada uma significativa perda de peso corporal, expressa em porcentagem, em relação ao momento zero, antes do início das provas (Tabela 2). Tais perdas se apresentaram de forma gradativa de acordo com a distância percorrida em cada prova. CARLSON (1985) e ROSE et al. (1979) relatam que o exercício de enduro tem como conseqüência marcante a perda de fluidos corporais decorrente da sudorese que está intimamente relacionada com a manutenção da temperatura corpórea do animal. A sudorese prolongada resulta em perdas de fluidos e eletrólitos dos compartimentos intra e extracelulares.

No entanto, quanto ao peso, não foi observada diferença entre os grupos experimentais no decorrer da prova de $30 \mathrm{~km}$, revelando que a administração da pasta eletrolítica não apresentou nenhum "benefício" durante as provas curtas e nas condições climáticas em que os animais foram avaliados. Na prova de $60 \mathrm{~km}$, observou-se diferença significativa quando se comparou o grupo controle no momento 1 em relação ao momento 3 e quando se comparou o grupo tratado nos momentos 1 e 2 em 
Tabela 1 - Temperatura e umidade relativa do ar em diferentes momentos $\left(\mathbf{M}_{0}, \mathbf{M}_{1}, \mathbf{M}_{2}\right.$ e $\left.\mathbf{M}_{3}\right)$ de provas de enduro realizadas em diferentes velocidades $\left(\mathrm{V} 10=10 \mathrm{~km} \mathrm{~h}^{-1} \mathrm{e} \mathrm{V} 15=15 \mathrm{~km} \mathrm{~h}^{-1}\right)$, com eqüinos que receberam ou não reposição eletrolítica.

\begin{tabular}{lccccc}
\hline \multicolumn{1}{r}{ Momento } & $\begin{array}{c}\text { Hora do } \\
\text { dia }\end{array}$ & Grupo & $\mathrm{n}$ & Temperatura ${ }^{\circ} \mathrm{C}$ & Umidade relativa (\%) \\
\hline $\mathrm{M}_{0}$ & & $\mathrm{~V} 10$ & 15 & 20,0 & 64 \\
(início) & $09: 00$ & $\mathrm{~V} 15$ & 18 & 26,3 & 67 \\
$\mathrm{M}_{1}$ & & $\mathrm{~V} 10$ & 15 & 27,8 & 40 \\
$(20 \mathrm{~km})$ & $12: 00$ & $\mathrm{~V} 15$ & 16 & 30,3 & 44 \\
$\mathrm{M}_{2}$ & & $\mathrm{~V} 10$ & 15 & 30,6 & 35 \\
$(30 \mathrm{~km})$ & $15: 00$ & $\mathrm{~V} 15$ & 11 & 32,3 & 36 \\
$\mathrm{M}_{3}$ & & & & 30,9 & 36 \\
$(60 \mathrm{~km})$ & $17: 00$ & $\mathrm{~V} 15$ & 11 & & 35 \\
\hline
\end{tabular}

relação ao momento 3. Foi observada uma menor porcentagem de perda de peso corporal no grupo tratado em relação ao grupo controle até o momento 2, corroborando o estudo de SCHOTT II et al. (1999). Nesse mesmo estudo, constatou-se que a suplementação com eletrólitos na forma de pasta oral hipertônica atenuou a perda de peso corporal, aumentando a ingestão voluntária de água por cavalos que realizaram uma prova de enduro de $60 \mathrm{~km}$ simulada em esteira. A extensão da prova por mais 30 quilômetros em condições climáticas mais estressantes (temperatura elevada e umidade relativa baixa) para o animal, promoveu uma significativa perda de peso corporal em relação ao momento anterior $\left(\mathrm{M}_{2}\right)$ em ambos os grupos.

Quando a velocidade do cavalo é mais lenta, perdas de fluidos e eletrólitos são menores ROSE et al. (1980) e SNOW et al. (1982). No repouso, cavalos de enduro tendem a ter valores de hematócrito menores tidos como reflexo de um maior volume plasmático desenvolvido como a resposta ao treinamento para prolongadas perdas de fluidos e estresse do calor CARLSON \& OCEN (1979). Foi observada essa tendência, no presente estudo, no decorrer dos 6 meses de treinamento entre as provas (dados não apresentados). Verificou-se também um aumento

Tabela 2 - Média \pm EPM das variáveis relacionadas de eqüinos, em diferentes momentos $\left(\mathbf{M}_{0}, \mathbf{M}_{1}, \mathbf{M}_{2}\right.$ e $\left.\mathbf{M}_{3}\right)$ de provas de enduro realizadas em diferentes velocidades $\left(\mathrm{V}_{10}=10 \mathrm{~km} \mathrm{~h}^{-1} \mathrm{e} \mathrm{V}_{15}=15 \mathrm{~km} \mathrm{~h}^{-1}\right.$ ), submetidos (grupo tratado=T) ou não (grupo controle=C) a reposição eletrolítica.

\begin{tabular}{|c|c|c|c|c|c|c|}
\hline Momento & Grupo & (n) & Peso (kg) & Perdas de peso $(\%)$ & Hematócrito (\%) & Prot. total $\left(\mathrm{g} \mathrm{dL}^{-1}\right)$ \\
\hline \multirow{4}{*}{$\begin{array}{l}\mathrm{M}_{0} \\
\text { (início) }\end{array}$} & $\mathrm{CV}_{10}$ & 7 & $410,0 \pm 13,5$ & $0,00 \pm 0,00 \mathrm{~A}$ & $37,7 \pm 0,5 \mathrm{~A}$ & $6,4 \pm 0,2 \mathrm{~A}$ \\
\hline & $\mathrm{TV}_{10}$ & 8 & $387,0 \pm 11,9$ & $0,00 \pm 0,00 \mathrm{~A}$ & $37,6 \pm 2,1 \mathrm{~A}$ & $6,5 \pm 0,1 \mathrm{~A}$ \\
\hline & $\mathrm{CV}_{15}$ & 10 & $404,0 \pm 13,5$ & $0,00 \pm 0,00 \mathrm{a}$ & $32,1 \pm 2,3 \mathrm{a}$ & $6,6 \pm 0,3 \mathrm{a}$ \\
\hline & $\mathrm{TV}_{15}$ & 8 & $388,0 \pm 12,0$ & $0,00 \pm 0,00 \mathrm{a}$ & $35,3 \pm 1,2 \mathrm{a}$ & $6,2 \pm 0,2 \mathrm{a}$ \\
\hline \multirow{4}{*}{$\begin{array}{l}M_{1} \\
(20 \mathrm{~km})\end{array}$} & $\mathrm{CV}_{10}$ & 7 & $391,5 \pm 12,5$ & $4,61 \pm 0,52$ В & $36,5 \pm 1,2 \mathrm{~A}$ & $7,0 \pm 0,3 \mathrm{~A}$ \\
\hline & $\mathrm{TV}_{10}$ & 8 & $362,2 \pm 12,6$ & $6,44 \pm 0,96$ В & $39,0 \pm 2,3 \mathrm{~A}$ & $7,2 \pm 0,2 \mathrm{~A}$ \\
\hline & $\mathrm{CV}_{15}$ & 9 & $383,5 \pm 14,1$ & $4,14 \pm 0,57 \mathrm{~b}$ & $47,5 \pm 1,2 \# \mathrm{~b}$ & $7,7 \pm 0,4 \mathrm{ab}$ \\
\hline & $\mathrm{TV}_{15}$ & 7 & $382,8 \pm 9,7$ & $3,15 \pm 0,76 b$ & $45,8 \pm 1,3 \mathrm{~b}$ & $7,4 \pm 0,2 \mathrm{~b}$ \\
\hline \multirow{4}{*}{$\begin{array}{l}M_{2} \\
(30 \mathrm{~km})\end{array}$} & $\mathrm{CV}_{10}$ & 7 & $387,0 \pm 13$ & $5,75 \pm 0,63 \mathrm{~B}$ & $42,5 \pm 1,1 \mathrm{~B}$ & $6,7 \pm 0,1 \mathrm{~A}$ \\
\hline & $\mathrm{TV}_{10}$ & 8 & $357,0 \pm 12,2$ & $7,78 \pm 1,10 \mathrm{~B}$ & $40,3 \pm 2,2 \mathrm{~A}$ & $6,8 \pm 0,2 \mathrm{~A}$ \\
\hline & $\mathrm{CV}_{15}$ & 6 & $382,2 \pm 20,6$ & $5,75 \pm 0,64 \mathrm{~b}$ & $45,0 \pm 1,1 \mathrm{~b}$ & $7,9 \pm 0,2 \# a b$ \\
\hline & $\mathrm{TV}_{15}$ & 5 & $381,0 \pm 11,9$ & $5,18 \pm 1,02 b$ & $45,0 \pm 1,2 \mathrm{~b}$ & $8,4 \pm 0,2 \mathrm{~b}$ \\
\hline \multirow{3}{*}{$\begin{array}{l}M_{3} \\
(60 \mathrm{~km})\end{array}$} & & & & & & \\
\hline & $\mathrm{CV}_{15}$ & 4 & $378,2 \pm 17,6$ & $8,01 \pm 1,03 \mathrm{c}$ & $44,33 \pm 0,9 \mathrm{~b}$ & $8,59 \pm 0,4 \mathrm{~b}$ \\
\hline & $\mathrm{TV}_{15}$ & 7 & $369,0 \pm 11,7$ & $8,13 \pm 0,63 \mathrm{c}$ & $48,80 \pm 2,8 \mathrm{~b}$ & $8,51 \pm 0,5 \mathrm{~b}$ \\
\hline
\end{tabular}


significativo do hematócrito (Tabela 2), a partir do segundo anel $\left(\mathrm{M}_{1}\right.$ para $\left.\mathrm{M}_{2}\right)$ na prova de $30 \mathrm{~km}$ e logo no primeiro anel na prova de $60 \mathrm{~km}\left(\mathrm{M}_{0}\right.$ para $\left.\mathrm{M}_{1}\right)$ mostrando a relação entre distância e intensidade de esforço aqui avaliados.

Os valores da concentração de proteínas totais (Tabela 2) apresentaram um comportamento semelhante ao dos valores de hematócrito. A desidratação severa durante o exercício de enduro causa uma marcante elevação na concentração de proteína plasmática (CARLSON, 1983). Esta desidratação promoveu um significativo aumento da concentração de proteínas totais na prova de $60 \mathrm{~km} \mathrm{a}$ partir do segundo anel $\left(\mathrm{M}_{2}\right) \mathrm{e}$, nesse mesmo momento, quando comparados os grupos controle das diferentes provas devido a um maior grau de esforço e estresse aos quais os animais foram submetidos. A administração da pasta eletrolítica também não apresentou nenhum efeito colateral aos animais submetidos ao esforço aqui avaliado.

Apesar de ocorrer um aumento significativo da frequência cardíaca a cada tempo de chegada para avaliação veterinária (tempo 120 e 210, referentes ao $\mathrm{M}_{1}$ e $\mathrm{M}_{2}$, respectivamente), quando comparados ao tempo zero do mesmo grupo, na prova de $30 \mathrm{~km}$ (Tabela $3)$, tais valores decresceram para valores iniciais, dentro dos primeiros 10 minutos de chegada, indicando que tais animais se mostravam condicionados para o referido esforço. Geralmente, um cavalo bem condicionado vai apresentar uma recuperação da frequiência cardíaca dentro dos primeiros 10 minutos da chegada. O prolongamento do período de recuperação indica desequilíbrio hidroeletrolítico, condicionamento inadequado, ou dor (FLAMÍNIO \& RUSH (1998). Nesta prova, de 30km, encontrou-se uma diferença significativa dos valores quando se comparou o grupo tratado com o grupo controle nos tempos 120 e 130 minutos, porém tal diferença deveuse ao fato de dois animais presentes no grupo tratado, excepcionalmente apresentarem freqüências cardíacas mais elevadas nestes tempos, possivelmente, devido ao estresse relacionado com a punção venosa, aferição cardíaca e resfriamento realizado por pessoal desconhecido para os animais, não havendo qualquer influência da utilização da pasta.

Na prova de $60 \mathrm{~km}$ (Tabela 4), percebeu-se uma tendência do grupo tratado apresentar valores de frequiência cardíaca mais baixos, quando comparados aos do grupo controle no decorrer da prova. A observação de FLAMÍNIO \& RUSH (1998), acima relatada, também foi observada nesta prova, revelando que o tempo de treinamento, bem como o condicionamento dos animais foi efetivo para tal esforço. HODGSON et al. (1993) relatam que o condicionamento melhora a habilidade do cavalo de eliminar calor pela evaporação do suor, pela pele e trato respiratório. Com isso, torna-se importante desenvolver um programa apropriado de treinamento e aclimatação para os climas quentes antes de um evento esportivo.

Os resultados de hemograma não apresentaram variação significativa durante o período no qual os animais foram submetidos ao treinamento nem durante as provas.

A administração oral da pasta eletrolítica, não trouxe nenhum efeito colateral aos cavalos em

Tabela 3 - Médias de freqüência cardíaca de eqüinos submetidos à prova de enduro de $30 \mathrm{~km}$ (grupo tratado) ou não (grupo controle) a reposição eletrolítica. Resultados expressos em média de batimentos por minuto, \pm erro padrão da média.

\begin{tabular}{|c|c|c|c|c|c|c|c|c|c|c|c|c|c|c|}
\hline \multirow{2}{*}{$\frac{\text { Momentos }}{\text { Tempo(min) }}$} & \multicolumn{3}{|l|}{$\mathrm{M}_{0}$} & \multicolumn{5}{|l|}{$\mathrm{M}_{1}$} & \multicolumn{6}{|l|}{$\mathrm{M}_{2}$} \\
\hline & 0 & 120 & 125 & 130 & 135 & 150 & 210 & 215 & 220 & 225 & 240 & $\mathrm{CV}$ & $\mathrm{F}$ & $\mathrm{P}$ \\
\hline controle & 36 & 47.43 & 43.7 & 38.14 & 40.9 & 40.9 & 54.3 & 46.3 & 40.1 & 41.4 & 41.4 & 11.38 & 9.90 & 0.0001 \\
\hline \multirow[t]{3}{*}{$(n=7)$} & \pm & \pm & \pm & \pm & \pm & \pm & \pm & \pm & \pm & \pm & \pm & & & \\
\hline & 0.98 & 2.5 & 2.24 & 1.03 & 1.3 & 1.3 & 3.04 & 2.16 & 1.77 & 1.62 & 1.62 & & & \\
\hline & $\mathrm{DE}$ & $\mathrm{AB}$ & $\mathrm{BCD}$ & $\mathrm{CDE}$ & BCDE & BCDE & A & $\mathrm{ABC}$ & BCDE & $\mathrm{BCD}$ & $\mathrm{BCD}$ & & & \\
\hline tratado & 35.4 & $60.2 *$ & 47.3 & $43.5 *$ & 40.3 & 40.3 & 60 & 50.3 & 42.8 & 39 & 39 & 12.03 & 21.36 & 0.0001 \\
\hline \multirow[t]{3}{*}{$(\mathrm{n}=8)$} & \pm & \pm & \pm & \pm & \pm & \pm & \pm & \pm & \pm & \pm & \pm & & & \\
\hline & 1.12 & 2.81 & 2.62 & 1.18 & 2.12 & 2.12 & 2.1 & 2.6 & 1.36 & 1.07 & 1.07 & & & \\
\hline & $\mathrm{DE}$ & A & $\mathrm{BC}$ & $\mathrm{BCD}$ & CDE & CDE & A & B & BCD & CDE & $\mathrm{CDE}$ & & & \\
\hline $\mathrm{CV}$ & 8.15 & 13.57 & 14.8 & 7.5 & 12.3 & 12.3 & 12.2 & 13.7 & 10.2 & 9.11 & 9.11 & & & \\
\hline $\mathrm{F}$ & 0.17 & 11.31 & 1.02 & 11.34 & 0.06 & 0.06 & 2.49 & 1.33 & 1.41 & 1.65 & 1.65 & & & \\
\hline $\mathrm{P}$ & NS & 0.005 & NS & 0.005 & NS & NS & NS & NS & NS & NS & NS & & & \\
\hline
\end{tabular}

* Indica média estatisticamente diferente $(\mathrm{P}<0,05)$ quando comparada ao respectivo controle no mesmo tempo.

Médias seguidas de letras diferentes em cada linha diferem entre si $(\mathrm{P}<0,05)$ no teste Tukey.

$\mathrm{CV}=$ Coeficiente de variância.

$\mathrm{F}=$ Resultado do teste $\mathrm{F}$ de análise de variância.

NS = Não significante.

Ciência Rural, v.34, n.5, set-out, 2004. 
Tabela 4 - Médias de freqüência cardíaca de eqüinos submetidos à prova de enduro de 60km (grupo tratado) ou não (grupo controle) a posição eletrolítica. Resultados expressos em média de batimentos por minuto, \pm erro padrão da média.

\begin{tabular}{|c|c|c|c|c|c|c|c|c|c|c|c|c|c|c|c|c|}
\hline \multirow{2}{*}{$\begin{array}{l}\text { Momentos } \\
\text { Tempo } \\
\text { (min.) }\end{array}$} & \multicolumn{2}{|l|}{$\mathrm{M}_{0}$} & \multicolumn{4}{|l|}{$\mathrm{M}_{1}$} & \multicolumn{4}{|l|}{$\mathrm{M}_{2}$} & \multicolumn{3}{|l|}{$\mathrm{M}_{3}$} & \multirow[b]{2}{*}{$\mathrm{CV}$} & \multirow[b]{2}{*}{$\mathrm{F}$} & \multirow[b]{2}{*}{$\mathrm{P}$} \\
\hline & 0 & 90 & 95 & 105 & 120 & 160 & 165 & 175 & 190 & 330 & 335 & 345 & 390 & & & \\
\hline \multirow{4}{*}{$\begin{array}{l}\text { controle } \\
(n=10)\end{array}$} & 38,20 & 75,11 & 64,33 & 62,33 & 62,22 & 70,67 & 60,33 & 53,67 & 51,20 & 70,50 & 65,00 & 62,75 & 54,50 & 17,64 & 6,65 & 0,0001 \\
\hline & \pm & \pm & \pm & \pm & \pm & \pm & \pm & \pm & \pm & \pm & \pm & \pm & \pm & & & \\
\hline & 1,38 & 4,15 & 4,16 & 4,92 & 5,02 & 1,43 & 2,03 & 3,42 & 2,94 & 7,14 & 6,24 & 3,42 & 1,50 & & & \\
\hline & A & $\mathrm{C}$ & $\mathrm{BC}$ & $\mathrm{BC}$ & $\mathrm{C}$ & BC & $\mathrm{BC}$ & $\mathrm{AB}$ & $\mathrm{AB}$ & BC & $\mathrm{BC}$ & BC & $\mathrm{ABC}$ & & & \\
\hline \multirow{4}{*}{$\begin{array}{l}\text { Tratado } \\
(\mathrm{n}=8)\end{array}$} & 40,75 & 72,29 & 65,86 & 54,14 & 53,67 & 66,00 & 65,40 & 51,00 & 52,40 & 67,60 & 63,20 & 57,60 & 50,00 & 18,68 & 4,5 & 0,0001 \\
\hline & \pm & \pm & \pm & \pm & \pm & \pm & \pm & \pm & \pm & \pm & \pm & \pm & \pm & & & \\
\hline & 1,13 & 4,44 & 3,83 & 3,49 & 5,50 & 5,10 & 3,74 & 2,83 & 2,56 & 4,96 & 7,17 & 7,98 & 4,98 & & & \\
\hline & A & $\mathrm{C}$ & $\mathrm{BC}$ & $\mathrm{ABC}$ & $\mathrm{ABC}$ & $\mathrm{BC}$ & $\mathrm{BC}$ & $\mathrm{ABC}$ & $\mathrm{ABC}$ & $\mathrm{BC}$ & $\mathrm{BC}$ & $\mathrm{ABC}$ & $\mathrm{AB}$ & & & \\
\hline $\mathrm{CV}$ & 9,91 & 16,45 & 17,73 & 21,61 & 24,59 & 11,73 & 10,68 & 14,37 & 11,90 & 18,22 & 22,85 & 23,73 & 16,62 & & & \\
\hline $\mathrm{F}$ & 1,90 & 0,21 & 0,07 & 1,64 & 1,26 & 0,92 & 1,57 & 0,34 & 0,09 & 0,12 & 0,03 & 0,29 & 0,60 & & & \\
\hline $\mathrm{P}$ & NS & NS & NS & NS & NS & NS & NS & NS & NS & NS & NS & NS & NS & & & \\
\hline
\end{tabular}

ambas as provas $(30 \mathrm{e} 60 \mathrm{~km})$. Entretanto, o fato de que dentre os 10 animais que iniciaram a prova de $60 \mathrm{~km} \mathrm{e}$ não receberam a referida pasta (grupo controle), apenas 4 completaram-na e aqueles que receberam a pasta $(8$ animais), 7 concluíram o percurso, sugere-se que este procedimento, administração de pasta eletrolítica, poderá contribuir para manutenção da homeostasia dos animais submetidos a esforços prolongados.

\section{CONCLUSÃO}

Apesar da revisão da literatura sobre a reposição hidroeletrolítica em eqüinos ser bastante extensa, constatou-se, na realização deste estudo, que muitos fatos ainda precisam ser esclarecidos para uma melhor compreensão das respostas fisiológicas que ocorrem no eqüino submetido ao exercício de longa duração e/ou distância. Concluiu-se que a metodologia utilizada foi bastante similar à que ocorre em provas de enduro nas referidas distâncias, agregando diversos fatores estressantes aos quais os cavalos são submetidos durante estas provas.

Estudos referentes a maiores graus de esforço, distância e velocidade tornam-se necessários para a continuidade da avaliação dos processos fisiológicos que ocorrem nos cavalos, durante competições eqüestres de elite.

\section{AGRADECIMENTOS}

À CAPES pela concessão de bolsa de estudo e toda a equipe de cavaleiros, amazonas, professores, funcionários, médicos veterinários residentes, amigos e colegas de Pós graduação da FCAV/UNESP-Jaboticabal.

\section{FONTES DE AQUISIÇÃO}

a - Farmogral, Farmácia de Manipulação, Brasília-DF.

b -Tubos siliconizados Vacutainerâ. Becton Dickinson Indústria Cirúrgica Ltda. Curitiba, PR.

c-CELM Companhia Equipadora de Laboratório Modernos. Barueri, SP.

d- Proc GLM - SAS v. 6.12, Cary - EUA.

\section{REFERÊNCIAS BIBLIOGRÁFICAS}

BURCH, G.E. Study of water and heat loss from the respiratory tract of man. Arch Intern Med, Chicago, v.76, p.308, 1945.

CARLSON, G.P. Medical problems associated with protracted heat and work stress in horses. Compend Cont Educ Pract Vet Suppl, v.7, p.542, 1985.

CARLSON, G.P. Thermoregulation and fluid balance in the exercising horse. In: SNOW, D.H.; PERSON, S.G.B.; ROSE, R.J. (Ed). Equine exercise physiology. Cambridge: Granta, 1983 p. 275-309.

CARLSON, G.P.; OCEN, P.O. Composition of equine sweat following exercise in high environmental temperatures and in response to intravenous epinephrine administration. $\mathbf{J}$ Equine Med Surg, Princeton, v.3, p.27, 1979.

FAN, L.C.R. et al. Anion gap no sangue venoso em eqüinos. Ciência Rural, Santa Maria, v.24, p.101-104, 1994.

FLAMÍNIO, M.J.B.; RUSH, B.R. Fluid and electrolyte balance in endurance horses. Vet Clin North Am Equine Pract, Philadelphia, v.14, p.147-158, 1998.

GREENLEAF, J.E. et al. Hypervolemia in men from fluid ingestion at rest and during exercise. Aviat Space Environ Med, Alexandria, v.69, p.374-386, 1998.

HARRIS, P.; SNOW, D.H. Plasma potassium and lactate 
concentrations in Thoroughbred horses during exercise of varying intensity. Equine Vet J, Newmarket, v.23, p.220$225,1992$.

HINTON, M. The biochemical and clinical of exhaustion in the horse. Vet Annu, Bristol, v.18, p.169-172, 1978.

HODGSON, D.R. et al. Dissipation of metabolic heat in the horse during exercise. J Appl Physiol, Bethesda, v.74, p.1161, 1993.

JOHNSON, P.J. Physiology of body fluids in the horse. Vet Clin North Am Equine Pract, Philadelphia, v.14, p.1-22, 1998.

KERR, M.G.; SNOW, D.H. Alterations in plasma protein and electrolytes in horses following the feeding of hay. Vet Rec, London, v.110, p.538-540, 1982.

KERR, M.G.; SNOW, D.H. Composition of sweat of the horse during prolonged epinephrine (adrenalin) infusion, heat exposure and exercise. Am J Vet Res, Schaumburg, v.44, p.1571-1577, 1983 .

MANSMANN, R.A. et al. Synchronous diaphragmatic flutter in the horse. J Am Vet Med Assoc, Schaumburg, v.165, p.265-270, 1974.

McCONAGHY, F.F. et al. Effects of two types of training on sweat composition. Equine Vet J Suppl, Newmarket, v.18, p.285-288, 1995.

McCONAGHY, F.F. Thermoregulation. In: HODGSON, D.R.; ROSE, R.J. (Ed). The athletic horse: principles and practice of equine sports medicine. Philadelphia : Saunders, 1994. p.181.

McCUTCHEON, L.J. et al. Sweating rate and sweat composition during exercise a nd recovery in ambient heat and humidity. Equine Vet J Suppl, Newmarket, v.20, p.153, 1995.

McCUTCHEON, L.J.; GEOR, R.J. Influence of trainingassociated thermoregulatory adaptations on sweating rate and sweat composition in Thoroughbred horses. J Sports Sci, London, v.14, p.347, 1996.

NIELSEN, B. et al. Thermoregulation in exercising man during dehydration and hyperhydration with water and saline. Int J Biometeorol, Heidelberg, v.15, p.195, 1971.

ROSE, R.J. et al. Plasma biochemistry in the horse during 3day event competition. Equine Vet J, Newmarket, v.12, p.132, 1980.

ROSE, R.J.; HENDRICKSON, D.K.; KNIGHT, P.K. Clinical exercise testing in the normal Thoroughbred racehorse. Aust Vet J, Brisbane, v.67, p.345, 1990.

ROSE, R.J.; ILKIW, J.E.; MARTIN, I.C.A. Blood-gas, acidbase and haematological values in horses during an endurance ride. Equine Vet J, Newmarket, v.11, p.56-59, 1979.

ROWEL, L.B. Cardiovascular adjustments to thermal stress. In: SHEPHERD, J.T.; ABBOUD, F.M. (Ed.). Handbook of physiology: the cardiovascular system, peripheral and organ blood flow. Bethesda : American Physiology Society, 1983. p.967.

SCHOTT II, H.C. et al. Effects of electrolyte and glycerol supplementation on recovery from endurance exercise. Equine Exercise Physiology 5. Equine Vet J Suppl, Newmarket, v.30, p.384-393, 1999.

SCHOTT II, H.C.; HINCHCLIFF, K.W. Fluids, electrolytes, and bicarbonate. Vet Clin North Am Equine Pract, Philadelphia, v.9, p.577-604, 1993.

SCHOTT II, H.C.; HINCHCLIFF, K.W. Treatments affecting fluid and electrolyte status during exercise. Vet Clin North Am Equine Pract, Philadelphia, v.14, p.175-204, 1998.

SNOW, D.H. et al. Alterations in blood, sweat, urine and muscle composition during prolonged exercise in the horse. Vet Rec, London, v.110, p.377-384, 1982

SOSA LÉON, L.A. Treatment of exercise-induced dehydration. Vet Clin North Am Equine Pract, Philadelphia, v.14, p.159173, 1998 